\title{
Concurrent Synaptic and Systems Memory Consolidation during Sleep
}

\author{
Laura Mascetti, ${ }^{1,2}$ Ariane Foret, ${ }^{1,2}$ Jessica Schrouff, ${ }^{1,2}$ Vincenzo Muto, ${ }^{1,2}$ Vinciane Dideberg, ${ }^{3}$ Evelyne Balteau, ${ }^{1,2}$ \\ Christian Degueldre, ${ }^{1,2}$ Christophe Phillips, ${ }^{1,2}$ André Luxen, ${ }^{1,2}$ Fabienne Collette, ${ }^{1,2}$ Vincent Bours, ${ }^{3}$ \\ and Pierre Maquet ${ }^{1,2}$ \\ ${ }^{1}$ Cyclotron Research Centre, University of Liège, 4000 Liège, Belgium, ${ }^{2}$ Walloon Excellence in Life Sciences and Biotechnology, 4000 Liège, Belgium, and \\ ${ }^{3}$ Department of Human Genetics, University Hospital Sart-Tilman, University of Liège, 4000 Liège, Belgium
}

\begin{abstract}
Memories are consolidated during sleep by two apparently antagonistic processes: (1) reinforcement of memory-specific cortical interactions and (2) homeostatic reduction in synaptic efficiency. Using fMRI, we assessed whether episodic memories are processed during sleep by either or both mechanisms, by comparing recollection before and after sleep. We probed whether LTP influences these processes by contrasting two groups of individuals prospectively recruited based on BDNF rs6265 (Val66Met) polymorphism. Between immediate retrieval and delayed testing scheduled after sleep, responses to recollection increased significantly more in Val/Val individuals than in Met carriers in parietal and occipital areas not previously engaged in retrieval, consistent with "systems-level consolidation." Responses also increased differentially between allelic groups in regions already activated before sleep but only in proportion to slow oscillation power, in keeping with "synaptic downscaling." Episodic memories seem processed at both synaptic and systemic levels during sleep by mechanisms involving LTP.
\end{abstract}

\section{Introduction}

The mechanisms by which sleep promotes memory consolidation remain unsettled. A first theory ("systems-level memory consolidation") assumes that hippocampal-dependent memories are reorganized and strengthened in large-scale distributed brain circuits during sleep (Schwindel and McNaughton, 2011). During sleep, hippocampal-neocortical interactions would progressively transfer the burden of memory from hippocampal-neocortical circuits to long-term neocortical stores (Frankland and Bontempi, 2005). Remote memory is potentially strengthened in neocortical circuits through LTP (Frankland et al., 2001). The cortical modules within which memories are reorganized are not yet clearly identified. Medial frontal cortex is suspected to participate in memory rearrangement in animals and humans (Gais et al., 2007; Takashima et al., 2009). However, cortical circuits that eventually store mnemonic information potentially involve other associative cortices [parietal cortex (Takashima et al., 2009)] and modality-specific cortices, namely for visual memories, temporo-occipital areas (Takashima et al., 2009).

\footnotetext{
Received Jan. 20, 2013; revised April 7, 2013; accepted May 9, 2013.

Author contributions: L.M., A.F., E.B., A.L., F.C., and P.M. designed research; L.M., A.F., V.M., C.D., and P.M. performed research; L.M., A.F., J.S., V.D., E.B., C.D., C.P., F.C., V.B., and P.M. analyzed data; L.M., A.F., V.M., A.L., F.C., and P.M. wrote the paper.

This research was supported by the Belgian National Fund for Scientific Research, the University of Liège, the Queen Elisabeth Medical Foundation, the Léon Fredericq Foundation, the Belgian Inter University Attraction Program, and the Walloon Excellence in Life Sciences and Biotechnology program.

The authors declare no competing financial interests.

Correspondence should be addressed to Dr. Pierre Maquet, Cyclotron Research Centre (B30), Allée du 6 Août, 8, 4000 Sart-Tilman, Liège, Belgium. E-mail: pmaquet@ulg.ac.be.

DOI:10.1523/JNEUROSCI.0284-13.2013

Copyright $\odot 2013$ the authors $\quad 0270-6474 / 13 / 3310182-09 \$ 15.00 / 0$
}

Another theory ("synaptic downscaling" hypothesis) assumes a progressive synaptic potentiation throughout the brain during wakefulness in relation to local neuronal activity (Tononi and Cirelli, 2006). Slow oscillations during subsequent non-rapid eye movement (NREM) sleep are generated in proportion to local neuronal work (Kattler et al., 1994) and learning (Huber et al., 2004) achieved during wakefulness, and their exponential decline during sleep would reflect the recalibration of synaptic efficacy (Vyazovskiy et al., 2009). Because synaptic downscaling during NREM sleep would differentially affect synapses depending on the potentiation accrued during wakefulness, the theory predicts that signal-to-noise selectively increases for responses to learned stimuli the following day (Tononi and Cirelli, 2006). The neurotrophin BDNF is released in response to neural activity (Mowla et al., 1999) and causally involved in LTP (Patterson et al., 1996) and the formation of long-term memory (Bekinschtein et al., 2008). BDNF expression during wakefulness modulates subsequent slow waves during sleep (Faraguna et al., 2008).

Here, we used fMRI, which is exquisitely sensitive to synaptic activity in a given brain area (Canals et al., 2009), to identify brain regions in which responses change as a result of synaptic potentiation, at each step of memory consolidation. We tested whether retrieval of episodic memories after sleep was consistent with either or both types of memory consolidation in humans. Therefore, these two theories differ in that synaptic homeostasis predicts a global decrease in synaptic strength after sleep, whereas systems-level consolidation assumes a selective increase in synaptic connectivity after sleep in memory-related brain circuits. Although synaptic downscaling entails local memory processing associated with NREM sleep slow waves, systemic consolidation results in a large-scale redistribution of memory traces within the 
A
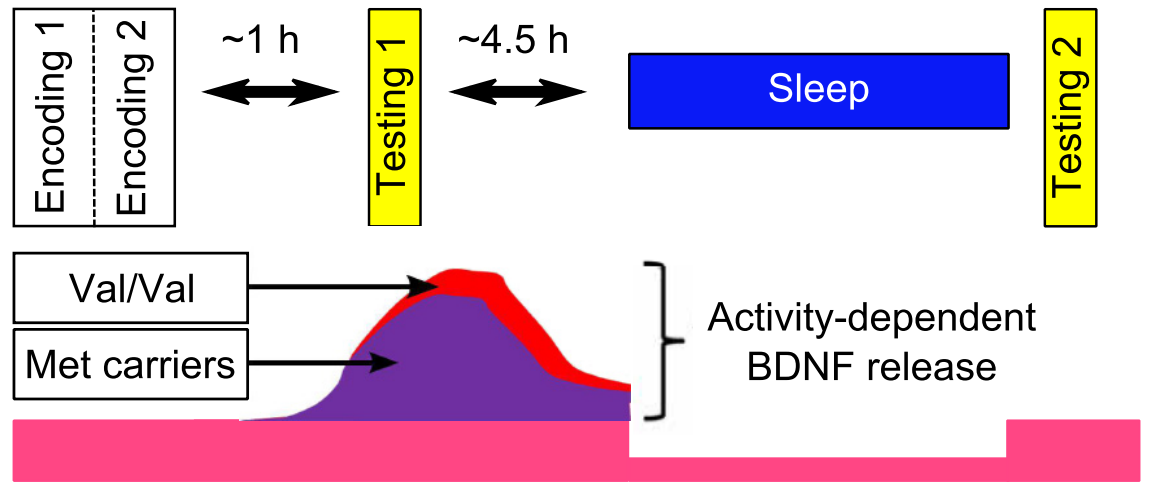

B

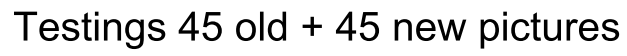

Encoding 135 pictures

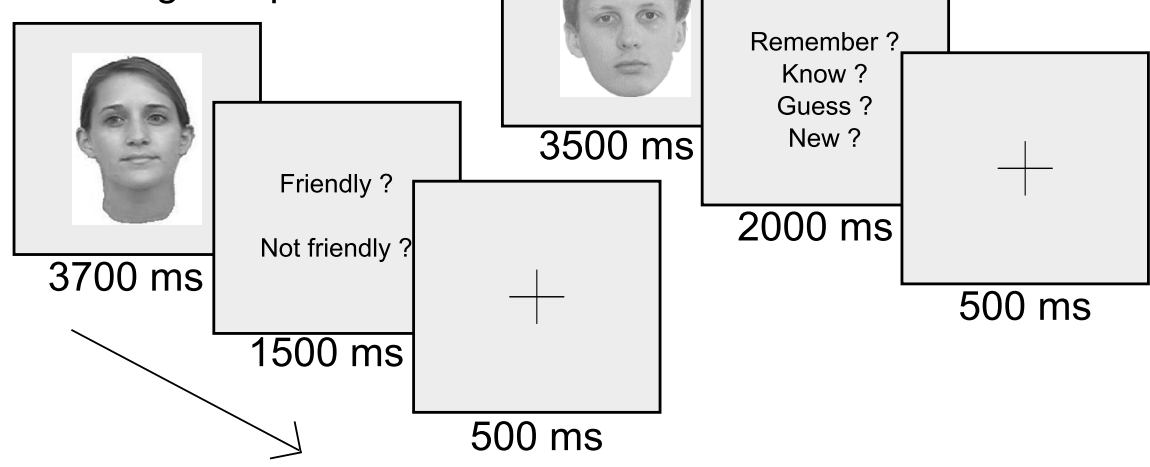

Figure 1. Experimental design. $\boldsymbol{A}$, The experiment includes three $\mathrm{fMRI}$ sessions: one encoding $(\times 2)$ and two retrieval sessions, initial and delayed, separated by a night of sleep (test night). The bottom schematically illustrates the release of BDNF in the two allelic groups. $\boldsymbol{B}$, Trial description for encoding and test sessions.

brain, including in areas that were not initially involved in retrieval. This leads to the prediction that memory retrieval after sleep should only be associated with changes in local responses in the case of synaptic downscaling. In contrast, if systemic consolidation takes place during sleep, memory consolidation should be associated with both local response changes and the recruitment of novel brain areas. Among the latter, we predicted that, in the current experiment, newly recruited cortical stores would include posterior associative cortices and occipital areas involved with face processing.

\section{Materials and Methods}

Moreover, to assess the involvement of LTP in human sleep-dependent memory consolidation, the experiment was based on the comparison of two groups of volunteers, prospectively recruited based on the Val66Met nonsynonymous single-nucleotide polymorphism of the BDNF gene (rs6265). Neurons transfected with the human A allele (Met-BDNF) showed a reduced activity-dependent BDNF release, and poorer episodic memory is observed in Met carriers (Egan et al., 2003). Therefore, by design, any difference in brain responses between groups would suggest implications of LTP in memory consolidation. We anticipated that Met carriers would induce less LTP than homozygous for the G allele (Val/Val) during encoding. The initial test session took place at 5:45 P.M., $\sim 1 \mathrm{~h}$ after encoding (Fig. $1 A$ ). In rodents, cortical BDNF infusion at encoding does not affect short-term retention but enhances long-term performance in a hippocampusdependent memory task (Alonso et al., 2005). We thus predicted that brain responses would not differ between allelic groups during initial retrieval but only during delayed testing after a period of sleep (scheduled between 10:30 P.M. and 7:00 A.M.). The next morning, delayed testing took place at $\sim 8: 30$
A.M., $\sim 1.5 \mathrm{~h}$ after awakening, after dissipation of sleep inertia. Although BDNF gene expression decreases during sleep (Cirelli and Tononi, 2000), we predicted that differential BDNF release during encoding and posttraining wakefulness (Fig. 1A) would result in enhanced slow oscillation during subsequent sleep in $\mathrm{Val} / \mathrm{Val}$ individuals relative to Met carriers (Faraguna et al., 2008). As a consequence, the synaptic downscaling hypothesis would predict that the signal-tonoise ratio during retrieval the next day, assessed as the difference between episodic recollection and recognition based on familiarity, should be particularly marked in Val/Val individuals in proportion to changes in slow oscillation induced by learning during posttraining sleep.

\section{Population}

Volunteers were recruited through advertisement on the University of Liège intranet. They gave written informed consent in compliance with procedures approved by the Ethics Committee of the Faculty of Medicine of the University of Liège. The absence of medical, traumatic, psychiatric, or sleep disorders was established by a semi-structured interview. Participants (males and females) were young (18-25 years old), healthy, right-handed, and lean (body mass index $<26)$ individuals. None complained about sleep disturbances as determined by the Pittsburgh Sleep Quality Index (PSQI) Questionnaire (score <6) (Buysse et al., 1989). None had worked night shifts during the preceding year or traveled through more than one time zone during the last 3 months. Extreme chronotypes were excluded according to the morningness-eveningness Horne-Ostberg (HO) Questionnaire (scores $<31$ or $>69$ ) (Horne and Ostberg, 1976). All participants had normal scores on the 21-item Beck Anxiety Inventory (BAI; score <11) (Beck et al., 1988) and the 21-item Beck Depression Inventory-II (BDI-II; score $<14$ ) (Steer et al., 1997). They were non-smokers and moderate caffeine and alcohol consumers. None was on medication, except for oral contraceptives. No caffeine was allowed during the experiment. Volunteers complying with these criteria performed the Raven's Progressive Matrices (Raven et al., 1983), and blood sample was obtained for BDNF genotyping (rs6265). Participants were eventually selected based on their BDNF genotype. Allelic groups were formed with participants matched according to sex, age, education level, chronotype, PSQI Questionnaire, and Raven scores (Table 1). They received a financial compensation for their blood test and their participation in the study.

\section{Genotyping}

Genomic DNA was extracted from blood samples using a MagNA Pure LC Instrument (Roche Applied Science). The DNA sequence of interest was amplified by PCR in a final volume of $50 \mu \mathrm{l}$ containing $0.6 \mu \mathrm{M}$ each primer (Thermo Fisher Scientific), $0.5 \mu$ l of Faststart TaqDNA Polymerase (Roche Diagnostics), $0.8 \mathrm{~mm}$ each deoxynucleotide triphosphate (Roche Diagnostics), and 20 ng of genomic DNA. After 10 min of denaturation at $95^{\circ} \mathrm{C}$, samples underwent 35 cycles consisting of denaturation $\left(95^{\circ} \mathrm{C}, 30 \mathrm{~s}\right)$, annealing $\left(60^{\circ} \mathrm{C}, 40 \mathrm{~s}\right)$, and extension $\left(72^{\circ} \mathrm{C}, 30 \mathrm{~s}\right)$, followed by a final extension of $7 \mathrm{~min}$ at $72^{\circ} \mathrm{C}$. These amplified DNA then underwent pyrosequencing reaction (Pyromark Q96 Vacuum Workstation, PSQ 96MA, Pyromark Gold Q96 Reagents; Qiagen). The sequences of the used primers are available on request.

\section{Experimental design}

The experiment consisted of an encoding session and two test sessions that took place $1 \mathrm{~h}$ after encoding and after a $16 \mathrm{~h}$ delay. During 1 week, volunteers were instructed to go to bed before midnight and sleep for at least $8 \mathrm{~h}$, 
Table 1. Demographic data in Val/Val and Met carriers

\begin{tabular}{|c|c|c|c|}
\hline & Val/Val & Met carriers & $p$ value \\
\hline Number of females & 8 & 8 & \\
\hline Number of males & 6 & 7 & \\
\hline Age & $21.7 \pm 1.6$ & $21.6 \pm 1.8$ & 0.81 \\
\hline HO Questionnaire & $54.7 \pm 5.4$ & $54.6 \pm 8$ & 0.98 \\
\hline PSQI Questionnaire & $2.9 \pm 1.1$ & $3.1 \pm 1.4$ & 0.77 \\
\hline $\mathrm{BAI}$ & $3.2 \pm 2.8$ & $4.5 \pm 2.5$ & 0.21 \\
\hline BDI-II & $2.9 \pm 3.5$ & $1.1 \pm 1$ & 0.35 \\
\hline Raven's progressive matrices: score (on 60 points) & $55.8 \pm 2.3$ & $55.8 \pm 2.2$ & 0.99 \\
\hline Raven's progressive matrices: testing time (min) & $28 \pm 9.1$ & $27.6 \pm 7.8$ & 0.90 \\
\hline
\end{tabular}

Except for the number of males and females, values correspond to the mean $\pm S D$.

and no naps were allowed [verified using sleep diaries and actigraphy (Actiwatch; Cambridge Neurotechnologies)]. Participants spent 3 nights under polysomnographic recording: (1) an adaptation night, (2) a baseline night after actigraphy and before the encoding session, and (3) a test night that took place between the initial and delayed testing (Fig. 1A).

\section{The memory task}

During the encoding session conducted in the MR scanner at 4:30 P.M., participants were asked to remember 135 pictures of neutral faces (67 females; no glasses, moustache, or beard; same size and luminance), each displayed during $3700 \mathrm{~ms}$ and presented in random order. A prompt then appeared and participants were instructed to specify within $1500 \mathrm{~ms}$ whether the face was friendly or not, with the help of a keyboard placed in their right hand. A fixation cross was displayed after the participant's response during $500 \mathrm{~ms}$. Null events consisting of the presentation of the fixation cross were randomly introduced and lasted at least $10,000 \mathrm{~ms}$. The encoding was repeated twice. Test sessions $(\sim 8 \mathrm{~min})$ took place at 5:45 P.M. (initial testing) and the next day at 8:30 A.M. (delayed testing). During each test session, participants had to make recognition memory judgments about 45 previously studied and 45 new pictures (equal sex ratio in both categories), presented in random order during $3500 \mathrm{~ms}$. The set of 45 "old" pictures differed between test sessions. Participants had up to $2000 \mathrm{~ms}$ to specify the following: (1) whether they were certain to have encoded the item and could retrieve specific details about the encoding episode ("remember" responses, recollection); (2) whether they were certain to have encoded the item but just had a feeling of familiarity ("know" responses); (3) whether they thought but were unsure that they had seen the face ("guess" responses, included to ensure a comprehensive assessment of familiarity measure); and (4) whether they thought the item had not been presented during encoding ("new" responses). They responded by a key press after which a fixation cross appeared for $500 \mathrm{~ms}$ (Fig. 1B). Null events also appeared randomly. Subjective [Karolinska sleepiness scale (KSS) (Akerstedt and Gillberg, 1990)] and objective [psychomotor vigilance task (PVT) (Dinges and Powell, 1985)] alertness was assessed before each $\mathrm{fMRI}$ session and after the last session of the first day. The memory task and the PVT were coded using MATLAB version 7.1 (MathWorks), with Cogent 2000 (version 1.25).

\section{fMRI data acquisition}

fMRI time series were acquired on a $3 \mathrm{~T}$ head-only scanner (Magnetom Allegra; Siemens Medical Solutions) operated with the standard transmitreceive quadrature head coil. Multislice $\mathrm{T} 2^{*}$-weighted functional images were acquired with a gradient EPI sequence using axial slice orientation and covering the whole brain/most of the brain (32 slices; FOV, $220 \times$ $220 \mathrm{~mm}^{2}$; voxel size, $3.4 \times 3.4 \times 3 \mathrm{~mm}^{3}$; $30 \%$ interslice gap; matrix size, $64 \times 64 \times 32$; TR, $2130 \mathrm{~ms}$; TE, $40 \mathrm{~ms}$; flip angle, $90^{\circ}$ ). The three initial volumes were discarded to avoid T1 saturation effects. A high-resolution T1-weighted image was also acquired for each subject [3D modified driven equilibrium Fourier transform (Deichmann et al., 2004): TR, 7.92 ms; TE, $2.4 \mathrm{~ms}$; TI, $910 \mathrm{~ms}$; flip angle, $15^{\circ}$; FOV, $256 \times 224 \times 176 \mathrm{~mm}^{3}$; $1 \mathrm{~mm}$ isotropic spatial resolution]. A mirror mounted on the standard head coil allowed individuals to see the stimuli presented on a screen placed at the rear of the scanner.

\section{EEG acquisition}

The adaptation night was recorded with a V-Amp amplifier (Brain Products). Six EEG (Fz, Cz, Pz, Oz, C3, and C4), four EOG (horizontal and vertical eye movements), and two chin EMG were recorded. A reference electrode was placed on the right mastoid. The other nights were recorded using a QuickAmp device (Brain Products) allowing for 64 EEGchannel recordings, two bipolar EOG, and one bipolar EMG. EEG was referenced to the mean of all electrodes. Electrode impedances were $<10$ $\mathrm{k} \Omega$. A $50 \mathrm{~Hz}$ notch filter was used, and the sampling rate was $500 \mathrm{~Hz}$ for both systems. Baseline night recordings began at 11:00 P.M. and ended at 7:00 A.M. Test night recordings began at 10:30 P.M., i.e., $\sim 6 \mathrm{~h}$ after encoding and $4.5 \mathrm{~h}$ after the end of the first test sessions. Volunteers were awakened at 7:00 A.M.

\section{EEG analyses}

EEG data were analyzed with the "fMRI Artifact Rejection and Sleep Scoring Toolbox" [FASST; http://www.montefiore.ulg.ac. be/ phillips/FASST.html (Leclercq et al., 2011)] and SPM8 (http://www.fil.ion.ucl.ac.uk/spm) implemented in MATLAB version 7.5. Sleep recordings were scored by two independent observers, using the criteria of the American Academy of Sleep Medicine (Iber and American Academy of Sleep Medicine, 2007), except for the use of $20 \mathrm{~s}$ windows. The final scoring was obtained after a consensus was achieved between scorers. Artifacts were identified manually and excluded from additional analyses. The first NREM sleep period, defined as the period between the first stage 2 and the first apparition of rapid eye movement (REM) sleep (first eye saccade), was extracted from the entire night. When REM sleep latency exceeded 90 min, suggesting that the first REM period had been "skipped," the first NREM period was ended after any sign of light NREM sleep or (even incomplete) REM sleep. We further split the first NREM episode in four quartiles and focused on the first quartile (Q1) because the increase in slow oscillation during posttraining sleep is known to be transient (Huber et al., 2004). Recordings were referenced to average mastoid.

Spectral analyses. Spectral analyses were conducted on the first NREM period and on Q1. For each electrode, power density was computed between 0.5 and $25 \mathrm{~Hz}$ using an FFT, $4 \mathrm{~s}$ windows overlapping by $2 \mathrm{~s}$, and a Hanning window (frequency resolution, $0.25 \mathrm{~Hz}$ ). EEG power was extracted for slow-wave activity (SWA; i.e., $0.75-4 \mathrm{~Hz}$ ) and EEG power centered on the spectral mode of slow oscillation $(0.75-1 \mathrm{~Hz})$, referred to as "slow oscillation power" (SOP).

Spindle detection. Spindle detection was based on amplitude detection parameters as defined by Mölle et al. (2002). In the implemented version [FASST (Leclercq et al., 2011)], three electrodes of reference were determined (i.e., $\mathrm{Fz}, \mathrm{Cz}$, and $\mathrm{Pz}$ or the closest if one was bad). The signal on these channels was then filtered between 8 and $20 \mathrm{~Hz}$. For each reference electrode, an amplitude criterion was then defined as the 95th percentile of the filtered signal in sleep stage 2 convolved with a rectangular window length of $100 \mathrm{~ms}$. Duration and distance criteria were the same for all reference channels and were set at 400 and $1000 \mathrm{~ms}$, respectively. The detected spindles were then epoched $(-300$ to $+1000 \mathrm{~ms})$ to perform a time-frequency decomposition between 11 and $16 \mathrm{~Hz}$ using Morlet waves. This operation allowed distinguishing between anterior or posterior spindles, depending on whether the largest power was observed on frontal or posterior channels, respectively. This detection was performed on the first NREM period. Finally, a density value (number of spindles per non-artifacted minute), as well as duration (milliseconds), amplitude (microvolts), and frequency (hertz) of spindles were extracted. The same reference electrodes were used for baseline and test nights.

\section{Statistical analyses}

$t$ tests were performed on questionnaire data, except for BDI-II, for which a $U$ test was performed because of the non-normal distribution of the data (significant at $p<0.05$ ). $t$ tests were performed on sleep durations, and midpoints measured by actigraphy (three recordings were discarded for technical reasons: $\mathrm{Val} / \mathrm{Val}, n=13$; Met carriers, $n=13$; normal distributions according to Kolmogorov-Smirnov test, significant at $p<0.05$ ). Concerning behavioral data, distributions were normal. Repeated-measures ANOVAs with session and genotypes within- and between-subject factors, 
respectively, were conducted on PVT [median, fastest (10th percentile) and slowest (90th percentile) reaction times] and KSS scores and separately on the number of "remembered hits" (RH), "known hits" (KH), and "guessed hits" (GH), "false alarms" (FA), "correct rejections" (CR), "misses" (MI), and discrimination index $\left(d^{\prime}\right)$ and criterion (Green and Swets, 1966) (significant at $p<0.05$ ). For $d^{\prime}$, planned comparisons were performed between genotypes and testings (Bonferroni's correction for four comparisons, $\alpha=$ $0.05 / 4$ : significant at $p<0.0125)$. Distributions of sleep stages were normal. Repeated-measures ANOVAs with night as within-subjects factor and genotype as between-subjects factor were conducted on durations and latencies of various sleep stages over the entire night, the first NREM cycle, and Q1 (significant at $p<0.05$ ). Concerning stage 2, stage 3, REM, and sleep duration of the entire night, values from five $\mathrm{Val} / \mathrm{Val}$ and one $\mathrm{Val} / \mathrm{Met}$ subjects were not available because of bad EEG signal in the end of the night. For spectral analysis, data were log-transformed because of non-normal distributions. Repeated-measures ANOVAs with night and electrodes as within-subjects factor and genotype as between-subjects factor were conducted, with Huynh-Feldt corrections for nonsphericity. Post hoc $t$ tests, Bonferroni's corrected for multiple comparisons, were performed to regionally specify EEG power changes between nights and genotypes (significant at $p<0.05$ ).

\section{fMRI data analyses}

fMRI data were analyzed using SPM8 implemented in MATLAB version 7.5. Functional scans were corrected for head motion, coregistered to the structural image, spatially normalized to a canonical EPI template conforming to the MNI, and spatially smoothed (Gaussian kernel, $8 \mathrm{~mm}$ FWHM). The analysis conformed to a mixed-effects analysis and accounted for fixed and random effects (RFX). For each subject, a general linear model was used to estimate brain responses at each voxel. Trials corresponding to $\mathrm{RH}, \mathrm{KH}, \mathrm{GH}, \mathrm{MI}, \mathrm{FA}$, and CR during encoding and test sessions were modeled as stick functions and convolved with the canonical hemodynamic response function. Movement parameters and a constant parameter were also included as covariates in the design matrix. High-pass filtering was implemented in the matrix design using a cutoff period of $128 \mathrm{~s}$ to remove slow drifts from the time series. Serial correlations in the fMRI signal were estimated using an autoregressive (order 1) plus white-noise model and a restricted maximum likelihood algorithm. Linear contrasts estimated the main effect of recollection $[\mathrm{RH}>(\mathrm{KH}+\mathrm{GH})]$ separately for each test session and for each encoding session [subsequent $\mathrm{RH}>(\mathrm{KH}+\mathrm{GH})$ ], as well as the session $\times$ recollection interaction (delayed $>$ initial testing). Summary statistic images corresponding to the interaction contrasts were smoothed (Gaussian kernel, $6 \mathrm{~mm}$ FWHM) and entered in the RFX analysis. Changes in SOP in Q1 (assessed as the first eigenvariate of the singular decomposition of significant changes in both groups in sleep EEG power between baseline and test nights), as well as spindle density during the first cycle of test night and changes in spindle density between first NREM cycles of baseline and test nights were included for each group as a regressor in the design matrix. RFX contrasts tested the effect of allelic group, which corresponded to a recollection $\times$ test $\times$ genotype interaction. Other contrasts assessed whether the overnight change in responses associated with recollection was linearly correlated to the increase in EEG power between nights or to spindle density during the first NREM cycle of test night or to changes in spindle density between first NREM cycles of baseline and test nights and differently so between allelic groups. A final contrast determined the brain responses associated with recollection separately for each test session. A correction for nonsphericity was applied to account for possibly unequal variance between groups. The resulting set of voxel values was thresholded at $p<$ 0.001 (uncorrected). Statistical inferences were performed at a threshold of $p<0.05$ after correction for multiple comparisons over small spherical volumes (10 $\mathrm{mm}$ radius), located in structures of interest reported in the literature, unless results were significant over the whole brain.

\section{Bayesian inferences and posterior probability maps}

In the RFX, we also computed posterior probability maps (PPMs) enabling conditional or Bayesian inferences about regionally specific effects (Friston and Penny, 2003). PPMs and effect size were computed for the contrast of recollection $[\mathrm{RH}>(\mathrm{KH}+\mathrm{GH})]$ to verify the absence of activation in regions during early and delayed testings.
Table 2. Behavioral data in Val/Val and Met carriers (mean \pm SD)

\begin{tabular}{|c|c|c|c|c|}
\hline & \multicolumn{4}{|c|}{ Number of trials (of 45) } \\
\hline & \multicolumn{2}{|l|}{ Initial testing } & \multicolumn{2}{|l|}{ Delayed testing } \\
\hline & $\mathrm{Val} / \mathrm{Val}(n=14)$ & $\begin{array}{l}\text { Met carriers } \\
(n=15)\end{array}$ & $\mathrm{Val} / \mathrm{Val}(n=14)$ & $\begin{array}{l}\text { Met carriers } \\
(n=15)\end{array}$ \\
\hline RH & $14.1 \pm 8.7$ & $11.5 \pm 4.9$ & $13.4 \pm 8.9$ & $13.1 \pm 4.7$ \\
\hline KH & $15.1 \pm 5.8$ & $15.6 \pm 5.6$ & $13.2 \pm 4.4$ & $11.0 \pm 4.4$ \\
\hline $\mathrm{GH}$ & $10.3 \pm 4.8$ & $11.3 \pm 3.2$ & $11.8 \pm 5.4$ & $13.4 \pm 4.2$ \\
\hline Ml & $5.4 \pm 4$ & $6.3 \pm 3.4$ & $6.5 \pm 4.4$ & $7.5 \pm 4.1$ \\
\hline$C R$ & $31.3 \pm 7.7$ & $28.1 \pm 4.7$ & $38.8 \pm 5.5$ & $35.3 \pm 5.3$ \\
\hline $\mathrm{FA}$ & $13.3 \pm 7.6$ & $16.3 \pm 4.7$ & $6 \pm 5.3$ & $9.7 \pm 5.3$ \\
\hline$d^{\prime}$ & $2.0 \pm 0.7$ & $1.5 \pm 0.4$ & $2.6 \pm 0.9$ & $1.9 \pm 0.5$ \\
\hline Criterion & $-0.4 \pm 0.6$ & $-0.4 \pm 0.3$ & $0.03 \pm 0.6$ & $-0.1 \pm 0.3$ \\
\hline
\end{tabular}

\section{Results}

\section{Demographic data}

Six hundred normal participants were screened in this study. One hundred thirty-four participants corresponded to the inclusion criteria and gave blood for BDNF genotyping. In this cohort, the studied single-nucleotide polymorphism was in Hardy-Weinberg equilibrium $\left(\chi^{2}=3.25, p=0.07\right)$ with genotypic frequency of 0.6 (G/G), 0.31 (G/A). and 0.09 (A/A).

Two allelic groups were eventually established: (1) $14 \mathrm{Val} / \mathrm{Val}$ (eight females) and (2) 15 Met carriers (eight females; six Met homozygous). Groups did not differ in terms of age, education level, chronotype, subjective sleep quality, anxiety, depression, and IQ (Raven matrices) (Table 1).

\section{Actigraphy}

During the week and especially during the $3 \mathrm{~d}$ preceding the experiment, participants were instructed to go to bed no later than 12:00 A.M. and to sleep for at least $8 \mathrm{~h}$. Over the last $3 \mathrm{~d}$, sleep duration (Val/Val, $9.22 \mathrm{~h} \pm 46 \mathrm{~min}$; Met carriers, $8.91 \mathrm{~h} \pm 44$ $\mathrm{min}$ ) and sleep midpoint (Val/Val, 4:08 A.M. $\pm 43 \mathrm{~min}$; Met carriers, 4:05 A.M. $\pm 40 \mathrm{~min})$ did not differ between groups $(t$ tests, $p=0.30$ and $p=0.86$, respectively; $n=13$ in each group).

\section{Alertness and episodic memory performance}

Subjective and objective alertness was measured before each scanning session and after the last session of the first day using KSS and PVT, respectively. Repeated-measures ANOVAs showed that they did not differ between groups $\left[\mathrm{KSS}, F_{(1,27)}=0.00, p=1\right.$; median reaction times at PVT, $F_{(1,27)}=0.03, p=0.86$; slowest reaction times at PVT (90th percentile), $F_{(1,27)}=0.00, p=0.99$; fastest reaction times at PVT (10th percentile), $\left.F_{(1,27)}=0.00, p=0.99\right]$.

Repeated-measures ANOVAs showed that recollection rates did not change significantly from initial to delayed testing $(\mathrm{RH}$ : genotype, $F_{(1,27)}=0.32, p=0.58$; testing, $F_{(1,27)}=0.28, p=0.60$; testing $\times$ genotype, $F_{(1,27)}=1.89, p=0.18$ ), whereas the number of familiarity-based responses decreased and the number of guess-based responses increased in both genotypes (KH: genotype, $F_{(1,27)}=0.25, p=0.62$; testing, $F_{(1,27)}=13.42, p=0.00$; testing $\times$ genotype, $F_{(1,27)}=2.42, p=0.13$; GH: genotype, $F_{(1,27)}=0.77, p=0.39$; testing, $F_{(1,27)}=6.98, p=0.01$; testing $\times$ genotype, $\left.F_{(1,27)}=0.18, p=0.68\right)$. MI rates showed a small but significant increase overnight, which did not differ between genotypes (MI: genotype, $F_{(1,27)}=0.47, p=0.50$; testing, $F_{(1,27)}=$ $4.19, p=0.05$; testing $\times$ genotype, $\left.F_{(1,27)}=0.00, p=0.96\right)$. FA rates decreased overnight particularly in $\mathrm{Val} / \mathrm{Val}$, although the change did not differ from Met carriers (FA: genotype, $F_{(1,27)}=$ $2.70, p=0.11$; testing, $F_{(1,27)}=90.06, p=0.00$; testing $\times$ genotype, $\left.F_{(1,27)}=0.27, p=0.61\right)$. CR rates increase to a larger extent 
Table 3. Sleep data for baseline and test nights in Val/Val and Met carriers (mean \pm SD)

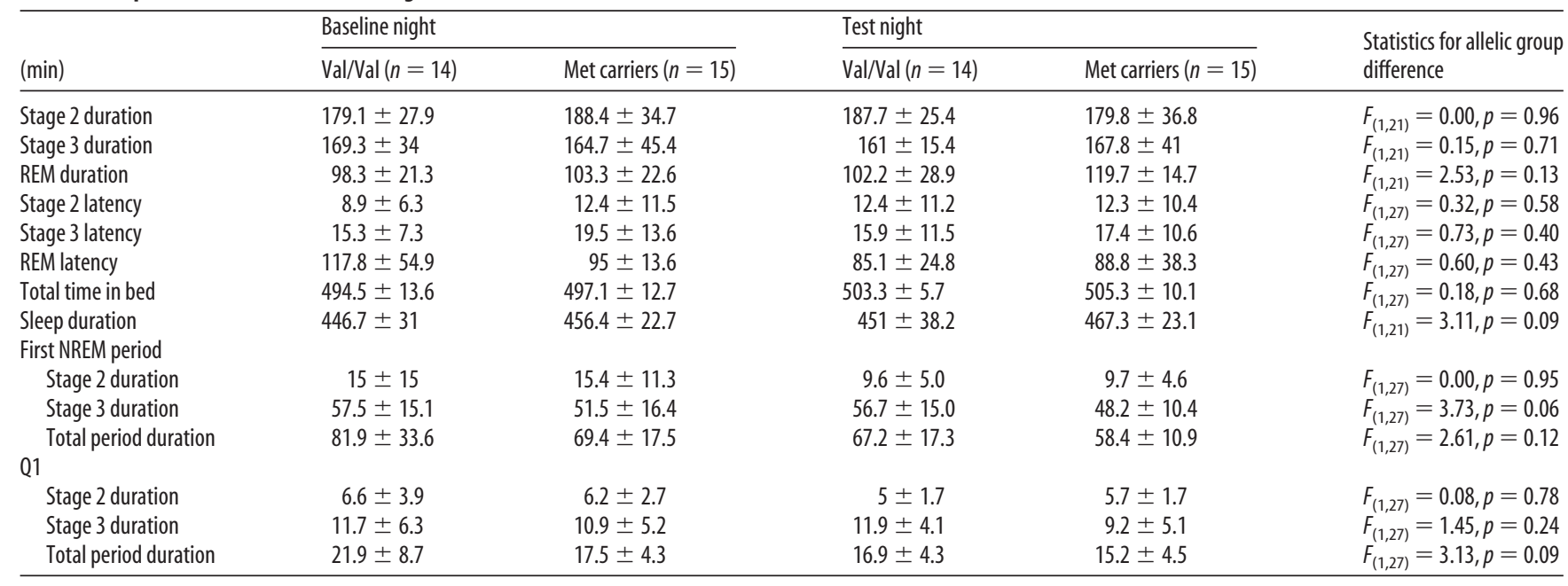

in $\mathrm{Val} / \mathrm{Val}$ individuals than in Met carriers, although this was not significant (CR: genotype, $F_{(1,27)}=2.68, p=0.11$; testing, $F_{(1,27)}=106.18, p=0.00$; testing $\times$ genotype, $F_{(1,27)}=0.04, p=$ $0.84)$. Discrimination index was larger in $\mathrm{Val} / \mathrm{Val}$ individuals than in Met carriers $\left(d^{\prime}\right.$, genotype, $\left.F_{(1,27)}=8.65, p=0.01\right)$ and changed to a larger, although not significant, extent in the former between test sessions (testing, $F_{(1,27)}=24.64, p=0.00$; testing $\times$ genotype, $F_{(1,27)}=1.29, p=0.27$ ) (Table 2). However, planned comparisons showed that $d^{\prime}$ did not differ between group during initial testing ( $p=0.016$, not significant after Bonferroni's correction for four comparisons). Both groups significantly increased $d^{\prime}$ from initial to delayed testing (Val/Val, $p=0.000$; Met carriers, $p=0.010$, Bonferroni's correction for four comparisons). These results show that both groups of normal participants benefit from sleep in terms of memory performance, in keeping with the literature (Gais et al., 2006). Importantly, the Val/Val group achieved significantly better discrimination than Met carriers during delayed testing ( $p=0.010$, Bonferroni's correction for four comparisons).

\section{Sleep data}

Sleep parameters during baseline and posttraining nights did not differ between allelic groups (Table 3 ).

Learning-dependent changes in NREM sleep are known to transiently occur during posttraining night and to predominate over frequencies corresponding to the slow oscillation $(<1 \mathrm{~Hz})$ (Huber et al., 2004). Therefore, we extracted the first NREM period from the entire night, as well as Q1. We checked that the duration of NREM sleep during the first NREM period and Q1 did not differ between groups (Table 3 ) and nights (repeated-measures ANOVAs, all $p$ values $>0.05$ ). We computed both SWA (NREM sleep EEG power between 0.75 and $4 \mathrm{~Hz}$ ) and SOP (NREM sleep EEG power between 0.75 and $1 \mathrm{~Hz}$, the spectral mode of slow oscillation) for each electrode over the first cycle and Q1.

Repeated-measures ANOVAs showed that SWA did not differ between nights or genotypes during the first NREM period (genotype, $F_{(1,27)}=0.23, p=0.64$; night, $F_{(1,27)}=1.44, p=0.24$; night $\times$ genotype, $\left.F_{(1,27)}=0.04, p=0.84\right)$. SWA tended to change differently between allelic groups during Q1 (genotype, $F_{(1,27)}=0.54, p=0.47$; night, $F_{(1,27)}=0.12, p=0.74$; night $\times$ genotype, $\left.F_{(1,27)}=4.11, p=0.053\right)$.

SOP showed a significant night $\times$ genotype interaction (genotype, $F_{(1,27)}=0.64, p=0.43$; night, $F_{(1,27)}=0.05, p=0.83$; night $\times$ genotype, $\left.F_{(1,27)}=4.53, p=0.04\right)$ during Q1 (Fig. $2 A$ ). In $\mathrm{Val} / \mathrm{Val}$ individuals, the average SOP increase over all electrodes was $6 \%$ between baseline and test nights. Post hoc $t$ tests (Bonferroni's correction) showed that power in Q1 increased significantly over $\mathrm{Fp} 2, \mathrm{FCz}, \mathrm{Cz}, \mathrm{POz}, \mathrm{Oz}, \mathrm{O} 1$, and $\mathrm{F} 3$ between baseline and test nights. The topography of the spectral changes is scarcely informative in the current study because of the widespread activation implied by explicit memory tasks. In Met carriers, SOP decreased on average by $5 \%$, although significant decreases were only detected over P8, PO8, C4, CP2, Oz, and PO7 ( $p$ values $<0.05$, Bonferroni's corrected; Fig. $2 B-D$ ). Confirmatory repeated-measures ANOVAs conducted separately in each allelic group confirmed a significant effect of night in $\mathrm{Val} / \mathrm{Val}$ individuals $\left(F_{(1,13)}=6.40, p=0.03\right)$ but not in Met carriers $\left(F_{(1,14)}=1.22, p=0.29\right)$. Consistent with our expectations, these results indicate that, between baseline and test nights, SOP increased in $\mathrm{Val} / \mathrm{Val}$ individuals and remained constant in Met carriers.

Finally, there was no difference between groups in spindle density, duration, amplitude, or frequency during the first NREM period ( $p$ values $>0.05)$.

\section{Brain responses activated in relation to recollection}

fMRI data showed that, across groups, initial recollection $[\mathrm{RH}>$ $(\mathrm{KH}+\mathrm{GH})]$ was associated with significant brain responses in a distributed set of areas in frontal, temporal, mesio-temporal, parietal, and cingulate cortices $\left(p_{\text {FWE-whole brain }}<0.05\right.$; Fig. $3 A$, yellow areas; Table 4). Initial recollection induced larger responses in the right intraparietal sulcus (IPS) in Met carriers than in the Val/Val group ( $p_{\mathrm{FWE} \text {-whole brain }}<0.05$; Fig. $3 A, B$, green area; Table 4), whereas no response was larger in the latter relative to the former. These results indicate that, if anything, recollection is increased with enhanced right parietal responses in Met carriers during initial testing. Based on animal data (Alonso et al., 2005), it is unlikely to result from differences in BDNF release since encoding.

From initial to delayed testing, responses elicited by recollection increased significantly more in the Val/Val group than in Met carriers in the left angular gyrus (AG), bilateral inferior occipital gyri (IOG), and bilateral IPS [ $p_{\mathrm{SVC}}<0.05$ (in which SVC is small volume correction); Fig. $3 A, B$, red areas; Table 4]. In contrast, no brain response was more enhanced in Met carriers during delayed relative to initial testing. To further dissect memory 

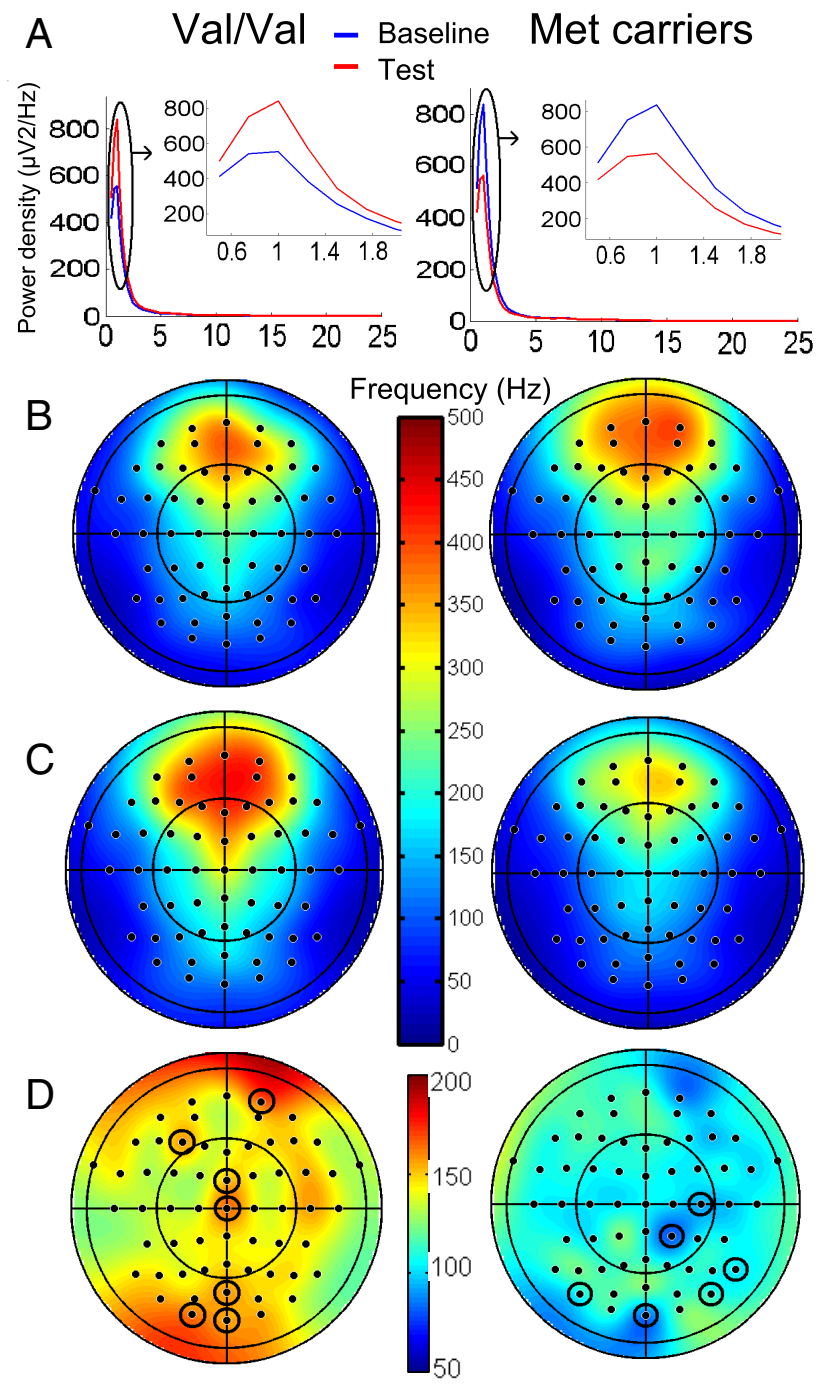

Figure 2. Sleep EEG spectral analysis. $\boldsymbol{A}$, Spectral density $(0.5-25 \mathrm{~Hz})$ on $\mathrm{Fp} 2$ (one of the electrodes that showed significant changes) during 01 in baseline (blue) and test nights (red). Insets, $0.5-2 \mathrm{~Hz}$. SOP increases between baseline and test nights in Val/Val individuals. Topography of SOP $(0.75-1 \mathrm{~Hz})$ during $\mathrm{Q} 1$ of baseline $(\boldsymbol{B})$ and test night (square microvolts) $(\boldsymbol{C})$. $\boldsymbol{D}$, Percentage between-night differences. Circled electrodes, $p<0.05$, Bonferroni's corrected. Left column, Val/Val; right column, Met carriers.

consolidation, we estimated the activity of these areas during early and delayed testing separately in each allelic group. Activity estimates showed that, in Val/Val individuals, left IOG and AG did not significantly respond to recollection during early testing ( $p>0.05$; Bayesian estimation of probability of activation $<4 \%$ in both regions). In contrast, their response became significant during delayed testing. None of these two areas overlapped with brain areas recruited during initial testing (left IOG did not even overlap with areas involved in encoding). This finding shows that these regions corresponded to newly recruited areas and suggests that systems-level memory consolidation took place overnight differentially between BDNF polymorphic groups. Conversely, responses in both IPS and right IOG significantly decreased between sessions in Met carriers. Only the right IPS was significantly recruited during early testing, as mentioned previously (Fig. $3 A, B$, area 6). Responses in left IPS and right IOG were not significant during early testing $(p>0.05$; Bayesian estimation of probability of activation $<5 \%$ in both regions) and responses were not significant in any of these three areas during delayed testing $(p>0.05$; Bayesian estimations of probability of activation of 0,9 , and $23 \%$ in left IPS, left IOG, and right IPS, respectively). Collectively, these results reveal a robust overnight response enhancement in $\mathrm{Val} / \mathrm{Val}$ individuals in left IOG and AG. In contrast, response changes in Met carriers occurred in areas that did not significantly respond during delayed retrieval.

fMRI data also showed that overnight changes in brain responses associated with recollection were linearly related to the changes in SOP during Q1 from baseline to test night in the right $\mathrm{mPFC}$ and left parahippocampal gyrus (PHG) in Val/Val but not Met carriers ( $p_{\mathrm{SVC}}<0.05$; Fig. $3 A, B$, blue areas; Table 4 ), both already activated in response to recollection during encoding and initial testing. Consistent with synaptic downscaling, these findings suggest that, in $\mathrm{Val} / \mathrm{Val}$, a local process takes place overnight in relation to the neuronal workload achieved during daytime (i.e., during learning, retrieval, or both) and in proportion to the gain in EEG power of slow oscillation during posttraining night. These results do not rule out an effect of systems-level consolidation: $\mathrm{mPFC}$ has been repeatedly involved in sleep-dependent hippocampo-dialog during sleep (Gais et al., 2007), and systemslevel consolidation was indirectly associated with slow waves, most likely through an entrainment of other NREM oscillations (e.g., spindles) (Sirota et al., 2003; Marshall et al., 2006). However, overnight changes in recollection-related responses were significantly related to neither spindle density during the first NREM cycle of test night nor change in spindle density during the first NREM cycle between baseline and test nights.

Finally, brain responses that significantly change between initial and delayed sessions in proportion to $d^{\prime}$ were looked for, but there was none.

\section{Discussion}

Memory consolidation was estimated using fMRI by comparing brain responses elicited by recollection [i.e., episodic recognition, $\mathrm{RH}>(\mathrm{KH}+\mathrm{GH})$ ] during two retrieval sessions scheduled $1 \mathrm{~h}$ after encoding and $16 \mathrm{~h}$ later, after a night of sleep, in two groups of normal participants differing by their Val66Met BDNF genotype. This contrast especially probes hippocampus-dependent memory (Yonelinas et al., 2005) and is particularly relevant for characterizing local or distributed processes of episodic memory retrieval.

Memory performance, estimated by $d^{\prime}$, improved after sleep in both allelic groups. Overall, Val/Val had better memory performance on both tests, although the difference became significant only after a night of sleep. This finding indicates that BDNF polymorphism influences overnight memory consolidation, suggesting that differential BDNF release induced by encoding triggers differential memory processing during subsequent hours. Note that changes in brain responses discussed below are independent of these behavioral results because they selectively probe episodic memory and contrast recollection to familiarity responses.

A transient increase in SOP was observed during Q1 in Val/ Val individuals, whereas no significant change was observed in Met carriers. Differences in sleep EEG spectrum are smaller than reported previously (Bachmann et al., 2012). In the current study, the objective was not to report the impact of Val66Met BDNF polymorphism on EEG rhythms but to use the functional consequence of this polymorphism as a tool to test hypotheses related to sleep-dependent memory consolidation. Stringent recruitment criteria matched allelic groups on a large number of demographic parameters, thereby attenuating possible differ- 
A
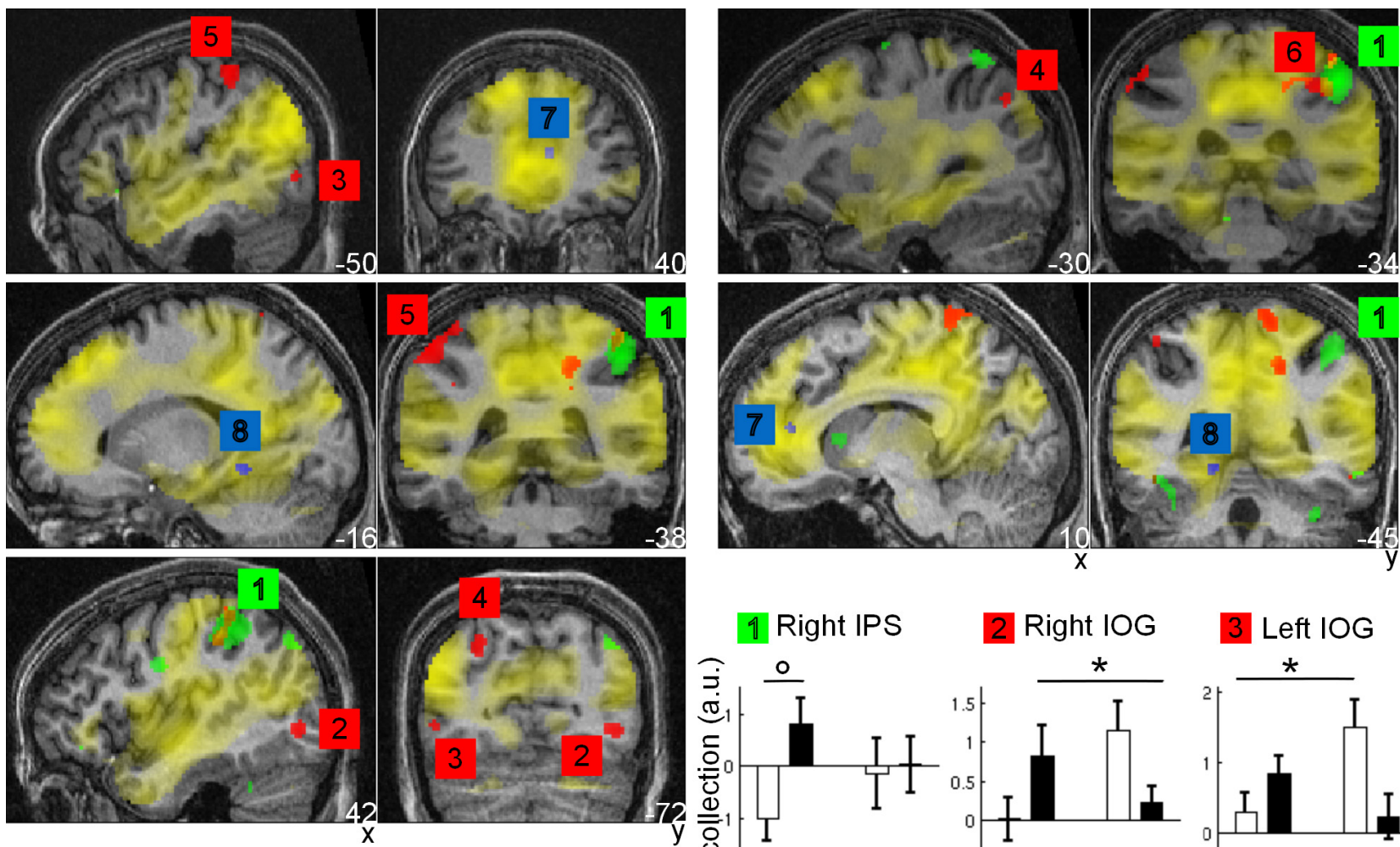

1 Right IPS
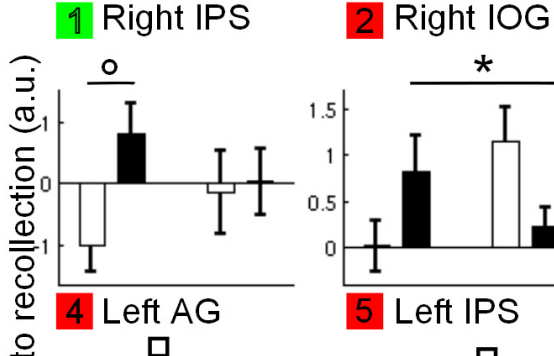

3 Left IOG

$\mathrm{B}$

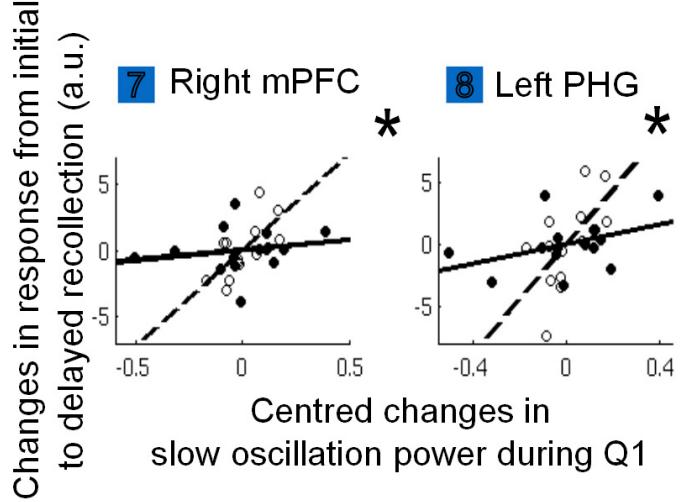

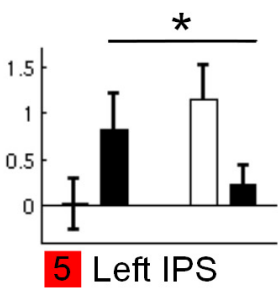
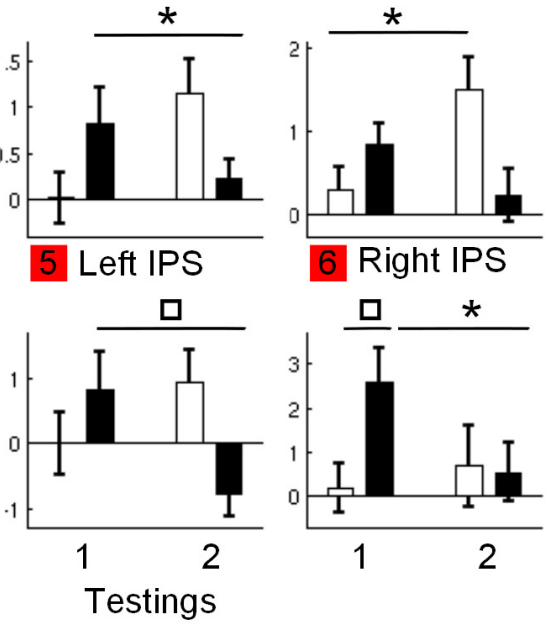

Figure 3. fMRI results. A, Yellow, Brain areas recruited during initial recollection in both groups. Green, Larger responses in Met carriers than in Val/Val during initial recollection. Red, Larger changes in response from initial to delayed recollection (Val/Val $>$ Met carriers). Blue, Areas in which changes in Val/Val are proportional to the changes in SOP from baseline to test night. Functional results are displayed at $p_{\text {uncorrected }}<0.001$, except for initial testing displayed at $p_{\text {uncorrected }}<0.05$ (to show all areas likely to be involved in early memory formation), over a typical individual MR image. $\boldsymbol{B}$, Parameter estimates (mean \pm SEM) of brain areas labeled as in $\boldsymbol{A}$. The two left bars pertain to initial testing (testing 1 ), and the two right bars pertain to delayed testing (testing 2 ). White bars, Val/Val; black bars, Met carriers. ${ }^{\circ} p_{\text {FWE-whole brain }}<0.05$ (1); ${ }^{*} p_{\text {SVc }}<0.05\left[2,3,6\right.$ (right bar), 7, 8]; ${ }^{\square} p_{\text {uncorrected }}<0.001[4,5,6$ (left bar)]. Scatter plots for areas 7 and 8 represent the regression between individual activity estimates and changes in SOP during Q1, assessed as the first eigenvariate of the singular decomposition of significant changes in both groups in sleep EEG power between baseline and test night. a.u., Arbitrary units. Open circles, dotted lines, Val/Val; filled circles, solid lines, Met carriers.

ences related to this polymorphism. In addition, a memory task is known to induce short-lasting changes in slow oscillation during sleep (Huber et al., 2004) and surely induces more subtle changes in EEG activity than total sleep deprivation (Bachmann et al., 2012). However, in keeping with our predictions, differential BDNF release induced before sleep by learning is associated with enhanced slow oscillations in $\mathrm{Val} / \mathrm{Val}$ individuals.

In $\mathrm{Val} / \mathrm{Val}$ individuals, responses induced by recollection increase between retrieval sessions in the right mPFC and left PHG in proportion to the increase in SOP recorded during Q1 during test night relative to baseline night. These brain areas share two important functional features. First, they were recruited before sleep, during encoding, and initial testing. Second, they are known to be systematically active in relation to slow waves during human NREM sleep (Dang-Vu et al., 2008). These elements concord with a local process, induced by learning and involving LTP, resulting in enhanced slow oscillation during sleep and an increase signal-to-noise response during later testing (i.e., increased response to recollection relative to familiarity-based responses). These results thus support the predictions of the downscaling hypothesis. They equally support any theory assuming the homeostatic regulation of local neuronal function in which the set parameter, instead of the average synaptic strength, would be a key functional parameter, such as redox state or oxidative metabolism (Scharf et al., 2008). Alternatively, one might assume a saturation of synaptic potentiation in $\mathrm{Val} / \mathrm{Val}$ individuals in the evening, explaining the lack of difference in memory-related responses during early retrieval. However, this hypothesis would not easily account for the difference in responses for remembered and known faces recorded the next morning, because synaptic downscaling would equally affect representation of remembered and known 


\begin{tabular}{|c|c|c|c|c|c|c|}
\hline & $x$ & $y$ & $z$ & Zscore & $p_{\text {FWE-whole brain }}$ & Reference SVC \\
\hline \multicolumn{7}{|c|}{$\mathrm{RH}>(\mathrm{KH}+\mathrm{GH})(\mathrm{Val} / \mathrm{Val}+$ Met carriers) (initial testing) } \\
\hline Left ventral mPFC & -8 & 44 & 4 & 7.543 & 0.000 & Whole brain \\
\hline Left superior frontal gyrus & -24 & 26 & 44 & 7.412 & 0.000 & Whole brain \\
\hline Left dorsal mPFC & -12 & 38 & 46 & 7.140 & 0.000 & Whole brain \\
\hline Right superior frontal gyrus & 22 & 34 & 48 & 5.626 & 0.001 & Whole brain \\
\hline Left posterior temporal sulcus & -52 & -70 & 24 & 7.221 & 0.000 & Whole brain \\
\hline Left inferior parietal lobule & -50 & -68 & 36 & 6.982 & 0.000 & Whole brain \\
\hline Left middle temporal gyrus & -58 & -14 & -14 & 6.529 & 0.000 & Whole brain \\
\hline Right mid-cingulate cortex & 8 & -28 & 42 & 7.131 & 0.000 & Whole brain \\
\hline Left posterior cingulate cortex & -8 & -48 & 28 & 7.028 & 0.000 & Whole brain \\
\hline Right posterior cingulate cortex & 12 & -48 & 32 & 6.645 & 0.000 & Whole brain \\
\hline Left cuneus & -10 & -90 & 22 & 6.579 & 0.000 & Whole brain \\
\hline Left retrosplenial cortex & -8 & -56 & 12 & 6.416 & 0.000 & Whole brain \\
\hline Right precentral cortex & 48 & -14 & 52 & 6.261 & 0.000 & Whole brain \\
\hline Left PHG & -24 & -26 & -18 & 6.179 & 0.000 & Whole brain \\
\hline Right superior parietal cortex & 28 & -42 & 64 & 5.580 & 0.001 & Whole brain \\
\hline Right retrosplenial cortex & 14 & -42 & 6 & 5.318 & 0.002 & Whole brain \\
\hline Left superior parietal cortex & -18 & -44 & 62 & 5.091 & 0.006 & Whole brain \\
\hline Right superior temporal sulcus & 54 & -46 & 22 & 6.241 & 0.000 & Whole brain \\
\hline Right middle temporal gyrus & 56 & -14 & -14 & 6.234 & 0.000 & Whole brain \\
\hline Right inferior parietal lobule & 66 & -38 & 26 & 5.850 & 0.000 & Whole brain \\
\hline Right PHG & 28 & -48 & -6 & 4.995 & 0.010 & Whole brain \\
\hline Right hippocampus & 28 & -18 & -20 & 4.929 & 0.013 & Whole brain \\
\hline \multicolumn{7}{|c|}{$\mathrm{RH}>(\mathrm{KH}+\mathrm{GH})$ (Met carriers $>$ Val/Val) (initial testing) } \\
\hline Right IPS & 48 & -34 & 48 & 5.02 & 0.008 & Whole brain \\
\hline \multicolumn{7}{|c|}{$\mathrm{RH}>(\mathrm{KH}+\mathrm{GH})(\mathrm{Val} / \mathrm{Val}>$ Met carriers) (delayed $>$ initial testing) } \\
\hline Right IOG & 42 & -74 & -2 & 3.620 & 0.007 & (Schiltz and Rossion, 2006) \\
\hline Left IOG & -48 & -70 & 0 & 3.140 & 0.026 & (Skinner et al., 2010) \\
\hline Left AG & -30 & -72 & 38 & 3.090 & 0.029 & (Vilberg and Rugg, 2007) \\
\hline Left IPS & -52 & -38 & 48 & 3.500 & 0.010 & (lidaka et al., 2006) \\
\hline Right IPS & 44 & -38 & 58 & 3.390 & 0.013 & (Yonelinas et al., 2005) \\
\hline \multicolumn{7}{|c|}{$\mathrm{RH}>(\mathrm{KH}+\mathrm{GH})(\mathrm{Val} / \mathrm{Val})$ (delayed $>$ initial testing) (SOP test $>$ baseline) } \\
\hline Right mPFC & 10 & 40 & 12 & 3.33 & 0.015 & (Sterpenich et al., 2007) \\
\hline Left PHG & -16 & -44 & -10 & 3.60 & 0.007 & (Yonelinas et al., 2005) \\
\hline
\end{tabular}

faces. Finally, the findings are not incompatible with systems-levels consolidation. The $\mathrm{mPFC}$ has been identified as a major area in the reorganization of episodic memories that integrates information and suppresses irrelevant representations from the hippocampus (Nieuwenhuis and Takashima, 2011). Repetitions of learned neural activity patterns during sleep were also associated with slow oscillation, although in the context of the temporal organization it imposes on other brain oscillations (Marshall and Born, 2007), especially spindles (Gais et al., 2002). Because we did not find any change in brain response that would regress with spindle activity, systems-level consolidation does not appear as the most likely mechanism to explain the findings in these two regions. Importantly, whichever the underlying mechanisms, our results further suggest that sleepdependent consolidation of episodic memories is modulated by cortical LTP.

Responses induced by recollection in left IOG and AG increased overnight only in $\mathrm{Val} / \mathrm{Val}$ individuals and significantly more so than in Met carriers. In $\mathrm{Val} / \mathrm{Val}$ individuals, the former region did not respond during encoding or initial testing and yet was recruited during delayed testing after sleep. This finding is incompatible with a local synaptic downscaling that would selectively process memories of the learned faces (synaptic homeostasis induced by previous neural work but unrelated to the probed memories remains possible but was not assessed here). In contrast, it supports the view of a systemslevel memory consolidation by which the burden of episodic memory not only would be redistributed in cortical memory stores but can even involve novel areas. The IOG is part of the face perception network and is especially involved in the identification of individuals
(Ishai, 2008). It is in good position to serve as a major long-term store for the face features encoded during the experiment. The AG did not show any response to recollection during initial retrieval but was recruited early on during encoding. In these conditions, the enhanced response observed during delayed testing is equally consistent with either a local or a distributed memory consolidation process.

Strictly speaking, the recruitment of these two areas during delayed testing might also result from the mere passage of time. However, volunteers spend 8 of $13.5 \mathrm{~h}$ in sleep between the end of encoding and their waking the next morning. In addition, it has been repeatedly shown that memory retention is enhanced by sleep compared with an equivalent period of wakefulness (Gais et al., 2006). Finally, BDNF infused in the cortex during the waking hours after encoding does not modify long-term memory in rodents (Alonso et al., 2005). Although a waking control group would theoretically settle this question, it appears that neither a sleep-deprived group nor a waking group tested during daytime are appropriate controls in the current instances because of confounds by either different sleep pressure or circadian phase. This issue deserves a future thorough experimental evaluation.

Finally, left IPS and right IOG did not respond to recollection during either initial or delayed testing, although their response significantly decreased between sessions in Met carriers. These findings do not contribute to support either theories of sleepdependent memory consolidation.

In conclusion, both sleep (Bachmann et al., 2012) and memory (Egan et al., 2003) are known to show genetically determined 
intersubject variability. The current results further reveal heretofore unsuspected interindividual variability in sleep-dependent memory processes at both the behavioral and cerebral levels. These findings warrant future assessment of genetically based variability of memory performance between participants in relation to sleep parameters.

More generally, our results provide experimental evidence that episodic memories are processed at both the local and distributed levels. They suggest that both synaptic downscaling and systems-level consolidation occur during sleep. Beyond their apparent antagonism, both processes likely contribute to generate robust episodic memories.

\section{References}

Akerstedt T, Gillberg M (1990) Subjective and objective sleepiness in the active individual. Int J Neurosci 52:29-37. CrossRef Medline

Alonso M, Bekinschtein P, Cammarota M, Vianna MR, Izquierdo I, Medina $\mathrm{JH}$ (2005) Endogenous BDNF is required for long-term memory formation in the rat parietal cortex. Learn Mem 12:504-510. CrossRef Medline

Bachmann V, Klein C, Bodenmann S, Schäfer N, Berger W, Brugger P, Landolt HP (2012) The BDNF Val66Met polymorphism modulates sleep intensity: EEG frequency- and state-specificity. Sleep 35:335-344. CrossRef Medline

Beck AT, Epstein N, Brown G, Steer RA (1988) An inventory for measuring clinical anxiety: psychometric properties. J Consult Clin Psychol 56:893-897. CrossRef Medline

Bekinschtein P, Cammarota M, Katche C, Slipczuk L, Rossato JI, Goldin A, Izquierdo I, Medina JH (2008) BDNF is essential to promote persistence of long-term memory storage. Proc Natl Acad Sci U S A 105:2711-2716. CrossRef Medline

Buysse DJ, Reynolds CF 3rd, Monk TH, Berman SR, Kupfer DJ (1989) The Pittsburgh Sleep Quality Index: a new instrument for psychiatric practice and research. Psychiatry Res 28:193-213. CrossRef Medline

Canals S, Beyerlein M, Merkle H, Logothetis NK (2009) Functional MRI evidence for LTP-induced neural network reorganization. Curr Biol 19: 398-403. CrossRef Medline

Cirelli C, Tononi G (2000) Differential expression of plasticity-related genes in waking and sleep and their regulation by the noradrenergic system. J Neurosci 20:9187-9194. Medline

Dang-Vu TT, Schabus M, Desseilles M, Albouy G, Boly M, Darsaud A, Gais S, Rauchs G, Sterpenich V, Vandewalle G, Carrier J, Moonen G, Balteau E, Degueldre C, Luxen A, Phillips C, Maquet P (2008) Spontaneous neural activity during human slow wave sleep. Proc Natl Acad Sci U S A 105: 15160-15165. CrossRef Medline

Deichmann R, Schwarzbauer C, Turner R (2004) Optimisation of the 3D MDEFT sequence for anatomical brain imaging: technical implications at 1.5 and 3 T. Neuroimage 21:757-767. CrossRef Medline

Dinges DF, Powell JW (1985) Microcomputer analysis of performance on a portable, simple visual RT task sustained operations. Behav Res Methods Instrum Comput 17:652-655. CrossRef

Egan MF, Kojima M, Callicott JH, Goldberg TE, Kolachana BS, Bertolino A, Zaitsev E, Gold B, Goldman D, Dean M, Lu B, Weinberger DR (2003) The BDNF val66met polymorphism affects activity-dependent secretion of BDNF and human memory and hippocampal function. Cell 112:257-269. CrossRef Medline

Faraguna U, Vyazovskiy VV, Nelson AB, Tononi G, Cirelli C (2008) A causal role for brain-derived neurotrophic factor in the homeostatic regulation of sleep. J Neurosci 28:4088-4095. CrossRef Medline

Frankland PW, Bontempi B (2005) The organization of recent and remote memories. Nat Rev 6:119-130. CrossRef Medline

Frankland PW, O'Brien C, Ohno M, Kirkwood A, Silva AJ (2001) AlphaCaMKII-dependent plasticity in the cortex is required for permanent memory. Nature 411:309-313. CrossRef Medline

Friston KJ, Penny W (2003) Posterior probability maps and SPMs. Neuroimage 19:1240-1249. CrossRef Medline

Gais S, Mölle M, Helms K, Born J (2002) Learning-dependent increases in sleep spindle density. J Neurosci 22:6830-6834. Medline

Gais S, Lucas B, Born J (2006) Sleep after learning aids memory recall. Learn Mem 13:259-262. CrossRef Medline

Gais S, Albouy G, Boly M, Dang-Vu TT, Darsaud A, Desseilles M, Rauchs G, Schabus M, Sterpenich V, Vandewalle G, Maquet P, Peigneux P (2007)
Sleep transforms the cerebral trace of declarative memories. Proc Natl Acad Sci U S A 104:18778-18783. CrossRef Medline

Green DM, Swets JA (1966) Signal detection theory and psychophysics. New York: Wiley.

Horne JA, Ostberg O (1976) A self-assessment questionnaire to determine morningness-eveningness in human circadian rhythms. Int J Chronobiol 4:97-110. Medline

Huber R, Ghilardi MF, Massimini M, Tononi G (2004) Local sleep and learning. Nature 430:78-81. CrossRef Medline

Iber C, American Academy of Sleep Medicine (2007) The AASM manual for the scoring of sleep and associated events: rules, terminology and technical specifications. Westchester, IL: American Academy of Sleep Medicine.

Iidaka T, Matsumoto A, Nogawa J, Yamamoto Y, Sadato N (2006) Frontoparietal network involved in successful retrieval from episodic memory. Spatial and temporal analyses using fMRI and ERP. Cereb Cortex 16: 1349-1360. Medline

Ishai A (2008) Let's face it: it's a cortical network. Neuroimage 40:415-419. CrossRef Medline

Kattler H, Dijk DJ, Borbély AA (1994) Effect of unilateral somatosensory stimulation prior to sleep on the sleep EEG in humans. J Sleep Res 3:159-164. CrossRef Medline

Leclercq Y, Schrouff J, Noirhomme Q, Maquet P, Phillips C (2011) fMRI artefact rejection and sleep scoring toolbox. Comput Intell Neurosci 2011:598206. CrossRef Medline

Marshall L, Born J (2007) The contribution of sleep to hippocampusdependent memory consolidation. Trends Cogn Sci 11:442-450. CrossRef Medline

Marshall L, Helgadóttir H, Mölle M, Born J (2006) Boosting slow oscillations during sleep potentiates memory. Nature 444:610-613. CrossRef Medline

Mölle M, Marshall L, Gais S, Born J (2002) Grouping of spindle activity during slow oscillations in human non-rapid eye movement sleep. J Neurosci 22:10941-10947. Medline

Mowla SJ, Pareek S, Farhadi HF, Petrecca K, Fawcett JP, Seidah NG, Morris SJ, Sossin WS, Murphy RA (1999) Differential sorting of nerve growth factor and brain-derived neurotrophic factor in hippocampal neurons. J Neurosci 19:2069-2080. Medline

Nieuwenhuis IL, Takashima A (2011) The role of the ventromedial prefrontal cortex in memory consolidation. Behav Brain Res 218:325-334. CrossRef Medline

Patterson SL, Abel T, Deuel TA, Martin KC, Rose JC, Kandel ER (1996) Recombinant BDNF rescues deficits in basal synaptic transmission and hippocampal LTP in BDNF knockout mice. Neuron 16:1137-1145. CrossRef Medline

Raven JC, Court JH, Raven J (1983) Manual for Raven's progressive matrices and vocabulary scales, revised edition. London: Lewis.

Scharf MT, Naidoo N, Zimmerman JE, Pack AI (2008) The energy hypothesis of sleep revisited. Prog Neurobiol 86:264-280. CrossRef Medline

Schiltz C, Rossion B (2006) Faces are represented holistically in the human occipito-temporal cortex. Neuroimage 32:1385-1394. CrossRef Medline

Schwindel CD, McNaughton BL (2011) Hippocampal-cortical interactions and the dynamics of memory trace reactivation. Prog Brain Res 193:163-177. CrossRef Medline

Sirota A, Csicsvari J, Buhl D, Buzsáki G (2003) Communication between neocortex and hippocampus during sleep in rodents. Proc Natl Acad Sci U S A 100:2065-2069. CrossRef Medline

Skinner EI, Grady CL, Fernandes MA (2010) Reactivation of contextspecific brain regions during retrieval. Neuropsychologia 48:156-164. CrossRef Medline

Steer RA, Ball R, Ranieri WF, Beck AT (1997) Further evidence for the construct validity of the Beck depression Inventory-II with psychiatric outpatients. Psychol Rep 80:443-446. CrossRef Medline

Sterpenich V, Albouy G, Boly M, Vandewalle G, Darsaud A, Balteau E, Dang-Vu TT, Desseilles M, D'Argembeau A, Gais S, Rauchs G, Schabus M, Degueldre C, Luxen A, Collette F, Maquet P (2007) Sleep-related hippocampo-cortical interplay during emotional memory recollection. PLoS Biol 5:e282. CrossRef Medline

Takashima A, Nieuwenhuis IL, Jensen O, Talamini LM, Rijpkema M, Fernández G (2009) Shift from hippocampal to neocortical centered retrieval network with consolidation. J Neurosci 29:10087-10093. CrossRef Medline 
Tononi G, Cirelli C (2006) Sleep function and synaptic homeostasis. Sleep Med Rev 10:49-62. CrossRef Medline

Vilberg KL, Rugg MD (2007) Dissociation of the neural correlates of recognition memory according to familiarity, recollection, and amount of recollected information. Neuropsychologia 45:2216-2225. CrossRef Medline
Vyazovskiy VV, Olcese U, Lazimy YM, Faraguna U, Esser SK, Williams JC, Cirelli C, Tononi G (2009) Cortical firing and sleep homeostasis. Neuron 63:865-878. CrossRef Medline

Yonelinas AP, Otten LJ, Shaw KN, Rugg MD (2005) Separating the brain regions involved in recollection and familiarity in recognition memory. J Neurosci 25:3002-3008. CrossRef Medline 